\title{
Editoriale
}

Che fare? Agli amici e colleghi tunisini

\section{Andrée Tabouret-Keller}

Traduttore: Stefano Corno

\section{(2) OpenEdition}

\section{Journals}

\section{Edizione digitale}

URL: http://journals.openedition.org/esp/476

DOI: 10.4000/esp.476

ISSN: 2532-0319

\section{Editore}

Centre d'Information sur l'Éducation Bilingue et Plurilingue

\section{Edizione cartacea}

Data di pubblicazione: 1 juin 2015

Paginazione: 2-3

ISSN: 1127-266X

\section{Notizia bibliografica digitale}

Andrée Tabouret-Keller, «Editoriale», Éducation et sociétés plurilingues [Online], 38 | 2015, Messo online

il 01 mars 2016, consultato il 25 février 2021. URL: http://journals.openedition.org/esp/476 ; DOl:

https://doi.org/10.4000/esp.476 


\section{QUE FAIRE? AUX AMIS ET COLLÈGUES TUNISIENS. ÉDITORIAL}

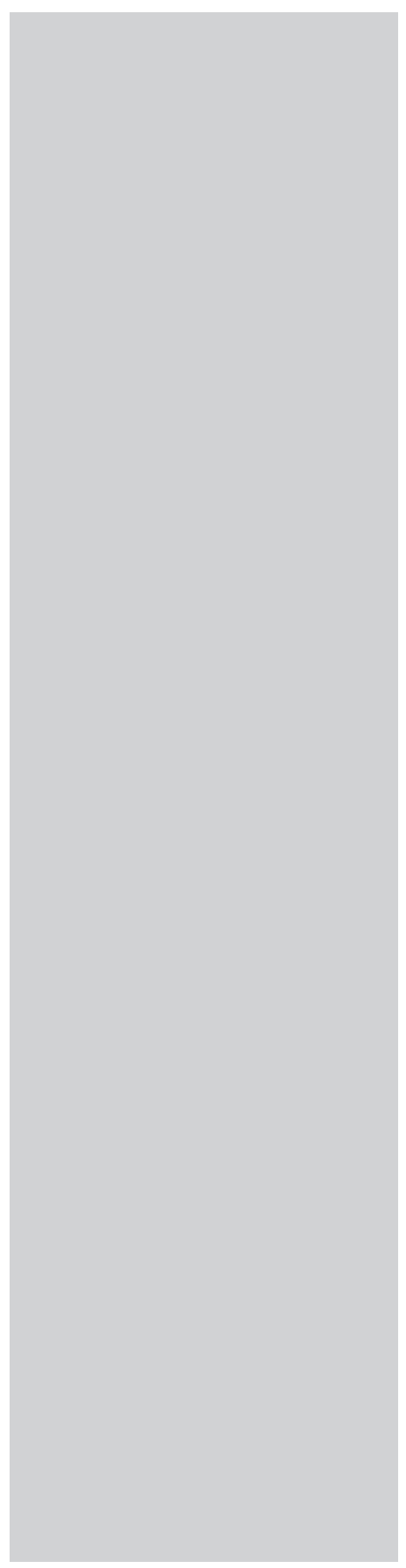

\section{Andrée TABOURET-KeLLER}

$\mathrm{O}^{1}$

omment passer sous silence les évènements qui nous ont pris à la fois dans une surprise totale et avec un frémissement d'horreur en France, à Paris, en ce début janvier et ces jours derniers en Tunisie, à Tunis? Ce n'est pas dans notre petit journal que nous pouvons faire l'analyse des conditions éminemment complexes qui ont abouti aux extrémités de ces assassinats. Mais en tant que parents, en tant que citoyens, en tant qu'enseignants, nous le sommes presque tous, nous ne pouvons pas éluder la question Que faire? En tant que collaborateurs d'Éducation et sociétés plurilingues, nos objectifs sont plus particulièrement de relier les questions relatives aux exigences des sociétés plurilingues, et plus particulièrement de l'école bi- et plurilingue, aux contraintes et aux libertés - linguistiques, politiques, culturelles - que chaque société connait face à l'éducation et à la formation de ses enfants et de sa jeunesse. Ce projet nous met face à l'une des difficultés qui se sont manifestées lors des évènements qui viennent d'être mentionnés, sans doute la principale: le déni de tolérance vis-à-vis de ce qui ne serait pas «pur», de ce qui serait mélangé, que ce soit du côté des langues - c'est notre spécialité - ou bien du côté des cultures qui nous concernent autant. Le fanatisme extrême est l'aspect le plus terrible du défaut de tolérance, en particulier du côté des engagements religieux.

Les recettes générales sont connues: l'ouverture, c'est-à-dire la connaissance des modes de vie différents, est sans doute la principale. Chacun, chacune, devant sa classe, devant sa feuille de papier à écrire pour notre petite revue, doit trouver sa solution personnelle; les principes généraux sont indispensables mais pour être mis en œuvre ils exigent une application concrète qui appartient à chacun et dans laquelle il peut s'investir. Il n'est donc pas question d'énumérer de tels exemples, peut-être une petite histoire peut servir d'illustration.

Permettez-moi de raconter une petite histoire tunisienne. Cela se passait au début des années soixante-dix. Nous nous déplacions 
Que faire? aux amis et collègues tunisiens. Éditorial

A. TABOURET-KeLLER en voiture et voulions connaître «le sud» dont nous avions sans doute une vision bien simpliste: les dunes, les oasis. Nous voulions rejoindre Gabès en partant de Tozeur. Sur une route déserte que l'on nous avait déconseillée, du sable et encore du sable, nous voyons un petit garçon - avait-il dix ans? - dans sa blouse bleue d'écolier qui nous fait un signe timide: on s'arrête, il monte. «Où vas-tu?»: pas de réponse mais un simple geste de la main: tout droit. «Comment tu t'appelles?» Réponse claire à voix bien timbrée, bien articulée «Je m’appelle Mohamed» et «où vas-tu?». Pas de réponse: n'aurions-nous pas mieux fait de le laisser au bord de la route? Jusqu'au moment où il tapote le bras du conducteur et dit, tout aussi clairement «Ici est ma maison». Il descend, du sable de tout côté - nous sommes inquiets - mais il traverse la route sans hésiter et s'engage dans ce qui pour nous ressemble à des dunes. Un peu plus loin nous découvrons, au loin les hauts de quelques tentes. C'est donc là qu'il allait, là qu'était sa maison. Puissions-nous œuvrer pour que chaque enfant ait sa maison et qu'il en sache et le chemin et la nommer, fût-ce dans une langue non-usuelle pour lui.

\section{CHE FARE? AGLI AMICI E COLLEGHI TUNISINI. EDITORIALE}

\section{Andrée Tabouret-Keller}

$\mathrm{C}^{\mathrm{p}}$ ome passare sotto silenzio gli eventi che ci hanno colti completamente alla sprovvista ed allo stesso tempo ci hanno fatto fremere d'orrore in Francia, a Parigi, ai primi di gennaio e in questi ultimi giorni a Tunisi? Certo il nostro piccolo giornale non ci permette di fare un'analisi delle condizioni estremamente complesse che hanno condotto agli estremi di queste stragi. Ma in quanto genitori, in quanto cittadini, in quanto insegnanti, quasi tutti noi lo siamo, non possiamo eludere la domanda Che fare? In quanto collaboratori di Educazione e società plurilingui, i nostri obiettivi sono più peculiarmente quelli di collegare le questioni relative alle esigenze delle società plurilingui, e più particolarmente dell'insegnamento bi- e plurilingue, agli obblighi ed alle libertà - linguistiche, politiche, culturali - che ogni società conosce di fronte all'educazione e alla formazione dei propri figli e della gioventù. Questo progetto ci mette di fronte ad una delle difficoltà che si sono manifestate al momento degli avvenimenti 
Che fare? Agli amici e colleghi tunisini. Editoriale

A. TABOURET-KeLleR appena menzionati, probabilmente la principale: la negazione della tolleranza nei confronti di ciò che non sia "puro", di ciò che sia misto, sia per quanto concerne le lingue - che restano la nostra specialità - oppure sul piano delle culture, che ci riguardano allo stesso modo. Il fanatismo estremo è l'aspetto più terribile della mancanza di tolleranza, particolarmente sul lato degli impegni religiosi.

Le ricette generali sono note: l'apertura, vale a dire la conoscenza dei modi di vita diversi dai propri, è probabilmente la principale. Ognuno di noi, davanti alla propria classe, davanti al foglio di carta da scrivere per la nostra piccola rivista, deve trovare la propria soluzione personale, i principî generali sono indispensabili, ma per essere messi in pratica esigono un'applicazione concreta che appartiene a ciascuno di noi e nella quale ognuno può far valere il proprio impegno. Non si tratta quindi di elencare simili esempi, ma forse una storiella può fungere da illustrazione.

Permettetemi di raccontare una piccola storia tunisina. Risale all'inizio degli anni Settanta. Ci muovevamo in macchina e volevamo conoscere "il sud" di cui avevamo probabilmente una visione semplicistica: le dune, le oasi. Volevamo raggiungere Gabès partendo da Tozeur. Su una strada deserta che ci era stata sconsigliata vediamo un ragazzino - non avrà avuto neanche dieci anni - con una casacca blu da scolaretto, che ci fa un timido segno: ci fermiamo, sale. "Dove vai?": nessuna risposta, ma un semplice gesto della mano: diritto. "Come ti chiami?" Risposta chiara, con una voce dal bel timbro, ben articolata "Mi chiamo Mohamed". E "dove vai?": nessuna risposta. Non avremmo fatto meglio a lasciarlo sul ciglio della strada? Finché non tira il conducente per la giacca e dice, in modo altrettanto chiaro "Qui è casa mia". Scende, sabbia da ogni lato - siamo preoccupati - ma attraversa la strada senza indugi e s'incammina in ciò che a noi sembrano dune. Un po' più in là scopriamo in lontananza le cime di alcune tende. Andava dunque là, quella era la sua casa. Potessimo operare affinché ogni bambino avesse una casa di cui conoscesse sia il cammino che il nome, fosse pure in una lingua non usuale per lui!

Traduction: Stefano Corno 